\title{
Exploring Smart Grid and Data Center Interactions for Electric Power Load Balancing
}

\author{
Hao Wang ${ }^{\dagger}$, Jianwei Huang ${ }^{\dagger}$, Xiaojun Lin ${ }^{\ddagger}$, Hamed Mohsenian-Rad* \\ ${ }^{\dagger}$ Department of Information Engineering, The Chinese University of Hong Kong, Shatin, Hong Kong \\ ${ }^{\ddagger}$ Department of Electrical and Computer Engineering, Purdue University, West Lafayette, USA \\ ${ }^{*}$ Department of Electrical Engineering, University of California, Riverside, USA \\ †\{haowang, jwhuang\}@ie.cuhk.edu.hk, ‘linx@ecn.purdue.edu, *hamed@ee.ucr.edu
}

\begin{abstract}
The operation of a data center consumes a tremendous amount of electricity, and the energy cost accounts for a large portion of the data center's operation cost. This leads to a growing interest towards reducing the energy cost of data centers. One approach advocated in recent studies is to distribute the computation workload among multiple geographically dispersed data centers by exploiting the electricity price differences. However, the impact of load redistributions on the power grid is not well understood yet. This paper takes the first step towards tackling this important issue, by studying how the power grid can take advantage of the data center's load distribution proactively for the purpose of power load balancing. We model the interactions between power grid and data centers as a two-stage problem, where the power grid operator aims to balance the electric power load in the first stage, and the data centers seek to minimize their total energy cost in the second stage. We show that this two-stage problem is a bilevel program with an indefinite quadratic objective function, which cannot be solved efficiently using standard convex optimization algorithms. Therefore, we reformulate this bilevel optimization problem as a linear program with additional finite complementarity slackness conditions, and propose a branch and bound algorithm to attain the globally optimal solution. The simulation results demonstrate that our proposed scheme can improve the load balancing performance by around $12 \%$ in terms of the electric load index and reduce the energy cost of data centers by $46 \%$ on average.
\end{abstract}

\section{INTRODUCTION}

With the fast development of cloud computing services, it is common for a cloud service provider (e.g., Google, Microsoft, or Amazon) to build multiple geographically dispersed large data centers across the country. Each data center may include hundreds of thousands of servers, storage equipment, cooling facilities, and power transformers. The energy consumption and cost of a single data center hence can be very significant [1]. For example, Google revealed that its data centers continuously draw almost $260 \mathrm{MW}$ of power, which is more than what the Salt Lake City consumes [2]. This has motivated a lot of research toward optimizing the data center operations to reduce the total energy cost. For example, Qureshi et al. in [1] proposed a cost minimizing method to coordinate cloud computing workload with electricity price differences for minimizing the total energy cost of distributed data centers. Some recent results further studied the energy cost minimization problem with green renewable energy [3,4], data center demand response [5], temperature aware management [6], multi-electricity-market environment [7], and deregulated electricity price [8].

Due to the enormous energy consumption, data centers are expected to have a great influence on the operation of power grid [9]. However, most existing results focused on the study of data centers' energy minimization and cost mini- mization, without detailed analysis of the impact on the power grid. For example, when large data centers suddenly increase their energy consumption in low price regions, they may overload the grid, which can cause various problems, such as a regional or major blackout.

To make the power grid more reliable and intelligent, there has been an industry wide effort to build the new power infrastructure often known as the smart grid [10]. Smart grid is equipped with advanced communication technologies, and is able to integrate energy suppliers and users more effectively through two-way communications. For example, an energy supplier can send real-time price information to the smart meters of users, and the users can change energy consumption in response to the price changes. This can effectively coordinate demand with supply, and hence avoid the danger of power overload.

The above approach of dynamic electricity pricing for effective demand side management has been discussed extensively in the recent literature [11-15]. For example, in [11], Mohsenian-Rad et al. suggested scheduling household devices based on the predicted price to minimize the electricity cost. Mohsenian-Rad and Leon-Garcia in [12] considered the electricity cost sharing problem in a distributed manner. Nguyen et al. [13] proposed a game theoretic model in which an electricity provider dynamically updates the energy prices by considering the load profiles of the users to reduce the peak load. Chen et al. [14] also studied the residential electricity scheduling problem based on a Stackelberg game. Wong et al. in [15] designed a time-dependent price to incentivize users to shift power load so as to relieve stress during peak time. However, most of the existing results focused on the residential demand shifting over time without considering the demand management over space.

Motivated by the fact that data centers are often geographically distributed and have large energy demands, we investigate the geographical power load balancing problem via managing the energy consumption of data centers across both space and time. Figure 1 illustrates the system under consideration. The smart grid tries to achieve the power load balancing by altering the electricity consumption of data centers through dynamic pricing, and the data centers respond to the electricity prices and manage the computing workload assignments to minimize their total energy cost.
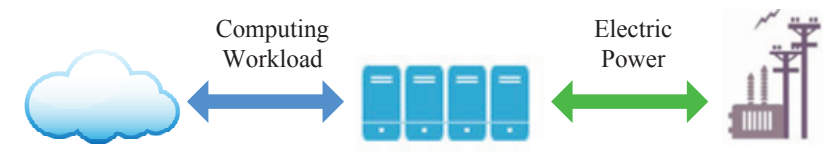

Cloud Computing

Data Centers

Smart Grid

Figure 1: Smart Grid and Data Center Interactions

The main contributions of this paper are listed as follows.

- To the best of our knowledge, this is the first paper that studies the interactions between smart grid and data centers by considering the active decisions on both sides. 
- We show that the two-stage problem is a bilevel program with an indefinite quadratic objective function, and cannot be solved by standard convex programming algorithms. We reformulate the two-stage problem as a linear program with complementarity slackness conditions, and design a branch and bound algorithm to attain the globally optimal solution.

- We also solve an integrated problem, and the solution provides a performance lowerbound to the two-stage problem. Simulation results show that the optimal solution of the two-stage problem is very close to the centralized optimal solution.

- Compared with the performance of LMP based pricing, our proposed method can both balance the power load for smart grid and reduce total energy cost for data centers, hence achieving a win-win result. Simulation results show that the load balancing performance of smart grid is improved by around $12 \%$ and the total energy cost of data centers is reduced by $46 \%$ on average.

The reminder of this paper is organized as follows. Next we formulate the system model in Section 2. In Section 3, we analyze the two-stage optimization problem and design a branch and bound algorithm to attain the global optimum. Performance of the proposed scheme is evaluated in Section 4. Finally, we conclude in Section 5.

\section{SYSTEM MODEL}

We consider a discrete time model $t \in \mathcal{T}=\{1, \ldots, T\}$, where the length of a time slot matches the time-scale at which the workload allocation decisions and dynamic pricing decisions can be updated (such as one hour $[7,16]$ ). Let $\mathcal{N}=$ $\{1, \ldots, N\}$ denote the set of geographically dispersed data centers, where each data center $i \in \mathcal{N}$ has $M_{i}$ homogeneous servers. As we explain later, not all the servers will be turned on during each time slot. Each data center is powered by a separate power substation in the power grid. We assume that there is a traffic aggregator responsible for distributing the total incoming computing workload $L^{t}$ within time slot $t$ to data centers in different regions [7]. We assume that there is no cost for distributing workload among data centers, but the QoS constraints of the workload assignment should be satisfied. Figure 2 illustrates the interactions between smart grid and data centers.

We assume that data centers cannot influence the electricity market price. Therefore, they are assumed to be price takers. We model the interactions between smart grid and data centers in two stages. Consider a time slot $t \in \mathcal{T}$.

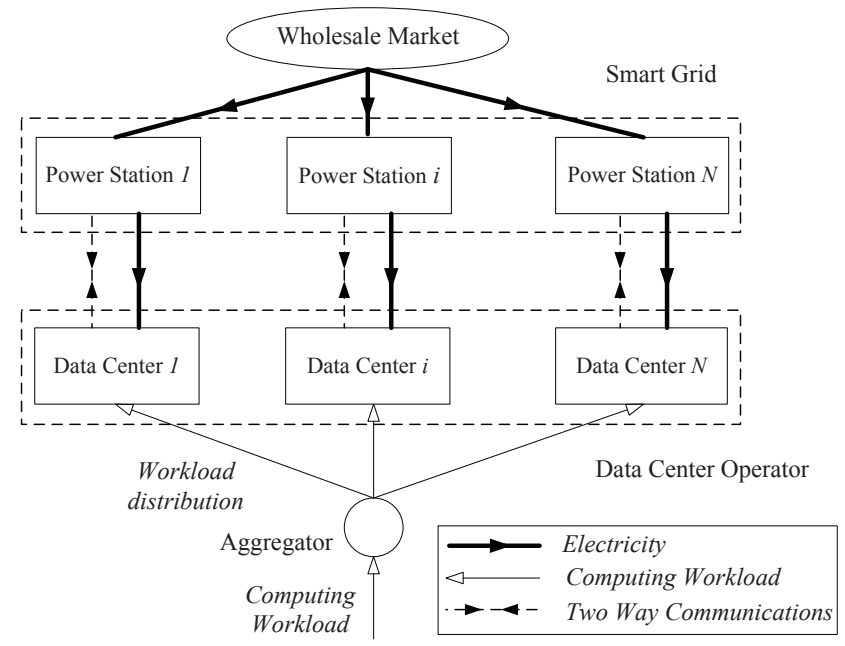

Figure 2: Architecture of data centers and smart grid
In Stage 1, the smart grid sets the charging threshold $s_{i}^{t}$ (which determines the electricity tariff) for each data center $i$ to balance the power load. In Stage 2, data centers cooperate with each other (as they belong to the same cloud computing provider) to minimize the total energy cost by determining the computing workload allocation $\lambda_{i}^{t}$ and the number of active servers $x_{i}^{t}$ in each data center $i$. Next we discuss these decisions in details.

\subsection{Stage 2: Data Center's Energy Cost Min- imization}

First, we consider the Stage-2 problem, where a cloud computing provider (such as Google) wants to minimize the total energy cost of multiple data centers. At time $t$, the smart grid charges the data center $i$ with the following regional electricity price $\pi_{i}^{t}$ (per unit of energy),

$$
\pi_{i}^{t}=\alpha_{i}^{t}+\beta_{i}\left(E_{i}^{t}-s_{i}^{t}\right),
$$

where $E_{i}^{t}$ is data center's the electricity consumption, $s_{i}^{t}$ is the charging threshold, $\beta_{i}>0$ is the sensitivity parameter, and $\alpha_{i}^{t}>0$ denotes the locational marginal price (LMP), all in the location $i$ at time $t$. Here $E_{i}^{t}$ is the decision variable by data center $i$ in Stage 2. The dynamic pricing scheme (1) is motivated by the tiered pricing which has been widely implemented in the power markets such as US, Japan, and China. The key idea is to set several tiers of energy consumption, and the unit price per unit of energy increases with the tiers progressively [17]. In (1), $s_{i}^{t}$ is the decision variable that is determined by the smart grid in Stage 1. The value of $s_{i}^{t}$ is assumed to be fixed and known in Stage 2 . The unit price $\pi_{i}^{t}$ will be lower than the LMP benchmark if the threshold $s_{i}^{t}$ is set to be larger than the energy consumption $E_{i}^{t}$.

In the following, we discuss the data centers' optimization constraints.

\subsubsection{Workload constraint}

In each time slot, the $N$ data centers should work together to complete the total workload of $L^{t}$ with the allocation to data center $i$ as $\lambda_{i}^{t}$.

$$
\sum_{i=1}^{N} \lambda_{i}^{t}=L^{t}, \lambda_{i}^{t} \geq 0, \forall i \in \mathcal{N}, t \in \mathcal{T} .
$$

\subsubsection{QoS (delay) constraint}

It is important for data centers to provide QoS guarantees to the users, and one important QoS metric is delay. We consider both the transmission delay (incured before the request arriving at the data centers) and the queuing delay (caused by the processing in the data centers). To model the transmission delay, we let $d_{i}^{t}$ denote the transmission delay experienced by a computing request from the aggregator to the data center $i$ during time slot $t$. Notice that $d_{i}^{t}$ is usually much less than the length of a time slot. To model the queuing delay, we use queuing theory to analyze the average processing time in data center $i$ when there are $x_{i}^{t}$ active servers processing workload $\lambda_{i}^{t}$ with a service rate $\mu$ per server, and the average waiting time is $\frac{1}{\mu x_{i}^{t}-\lambda_{i}^{t}}[3,7]$. To meet the QoS requirement, the total time delay experienced by a computing request should satisfy some delay bound $D$, which is the maximum waiting time that a request can tolerate. For simplicity, in this paper we will assume homogeneous requests that have the same delay bound $D$. Therefore, we have the following QoS constraint

$$
d_{i}^{t}+\frac{1}{\mu x_{i}^{t}-\lambda_{i}^{t}} \leq D, \forall i \in \mathcal{N}, t \in \mathcal{T},
$$

where $\mu x_{i}^{t} \geq \lambda_{i}^{t}$. 


\subsubsection{Server constraint}

At each data center $i$, there are tens of thousands of servers providing cloud computing services to meet users' requests. Let $M_{i}$ denote the maximum number of available servers. Since the number of servers is usually large, we can relax the integer constraint of number of active servers without significantly affecting the optimal result. Therefore, we have the following server constraint

$$
0 \leq x_{i}^{t} \leq M_{i}, \forall i \in \mathcal{N}, t \in \mathcal{T}
$$

\subsubsection{Energy consumption constraint}

The energy consumption of a data center mainly depends on its computing workload and the number of active servers. More precisely, the energy consumption of data center $i$ at time slot $t[1,9]$ is

$$
E_{i}^{t}=x_{i}^{t}\left(P_{\text {idle }}+\left(E_{\text {usage }}-1\right) P_{\text {peak }}\right)+x_{i}^{t}\left(P_{\text {peak }}-P_{\text {idle }}\right) \gamma_{i}^{t}+\xi
$$

where $P_{\text {idle }}$ and $P_{\text {peak }}$ represent the average idle power and average peak power of a single server, respectively. Power usage effectiveness (PUE), denoted by $E_{\text {usage, }}$, measures the energy efficiency of the data center, and is defined as the ratio of the data center's total energy consumption to the energy consumption of servers. Average server utilization of data center $i$ at time $t$, denoted by $\gamma_{i}^{t}$, is represented as $\gamma_{i}^{t}=\lambda_{i}^{t} /\left(\mu x_{i}^{t}\right)$. The parameter $\xi$ is an empirical constant [9]. We observe that the term $x_{i}^{t}\left(P_{\text {idle }}+\left(E_{\text {usage }}-1\right) P_{\text {peak }}\right)$ represents the base energy consumption, which only depends on the number of active servers. The term $x_{i}^{t}\left(P_{\text {peak }}-P_{\text {idle }}\right) \gamma_{i}^{t}$ represents the incremental consumption, which depends on the workload. We can rewrite $E_{i}^{t}$ in the equivalent form as

$$
E_{i}^{t}=a \lambda_{i}^{t}+b x_{i}^{t}+c, \forall i \in \mathcal{N}, t \in \mathcal{T},
$$

which is an affine function in terms of the number of active servers $x_{i}^{t}$ and the computing workload $\lambda_{i}^{t}$. The coefficients are $a=\left(P_{\text {peak }}-P_{\text {idle }}\right) / \mu, b=P_{\text {idle }}+\left(E_{\text {usage }}-1\right) \times P_{\text {peak }}$, and $c=\xi$.

Note that a data center $i$ needs to share the power supply with the background consumption, i.e., electricity usage by other industrial and residential users, in the same location. Since the total power supply capacity is limited at this station and the background load is time varying, then we limit the maximum power that can be consumed by data center $i$ at time $t$ as

$$
E_{i}^{t} \leq q_{i}^{t}, \forall i \in \mathcal{N}, t \in \mathcal{T}
$$

where $q_{i}^{t}$ denotes the available power supply to data center $i$ at time $t$.

With the above constraints, we can formulate the cloud computing provider's energy cost minimization problem in Stage 2. The objective is to minimize the data centers' total energy cost over all locations and all time slots by choosing the workload allocation $\lambda_{i}^{t}$ and the number of active servers $x_{i}^{t}$ for each data center $i \in \mathcal{N}$ and each time $t \in \mathcal{T}$. The Stage 2 problem is as follows.

Stage 2: Total energy cost minimization

$$
\min _{\boldsymbol{\lambda}, \boldsymbol{x}} E C o s t=\sum_{t \in \mathcal{T}} \sum_{i \in \mathcal{N}}\left(\alpha_{i}^{t}+\beta_{i}\left(E_{i}^{t}-s_{i}^{t}\right)\right) E_{i}^{t}
$$

subject to Constraints $(2)-(6)$.

Here the workload allocation vector $\boldsymbol{\lambda}=\left(\boldsymbol{\lambda}_{i}, \forall i \in \mathcal{N}\right)$, where $\boldsymbol{\lambda}_{i}=\left(\lambda_{i}^{t}, \forall t \in \mathcal{T}\right)$. The active server number vector $\boldsymbol{x}=$ $\left(\boldsymbol{x}_{i}, \forall i \in \mathcal{N}\right)$, where $\boldsymbol{x}_{i}=\left(x_{i}^{t}, \forall t \in \mathcal{T}\right)$.

Notice that the optimal values of $\boldsymbol{\lambda}$ and $\boldsymbol{x}$ of Problem (PS2) are functions of the charging thresholds $s=\left(s_{i}^{t}, \forall i \in\right.$ $\mathcal{N}, \forall t \in \mathcal{T})$.

\subsection{Stage 1: Smart Grid's Power Load Bal- ancing}

Now we consider the Stage- 1 power load balancing problem for the smart grid. Overload has been a major problem in traditional power grid, as it is often difficult to prevent users in a specific region to generate an excessive power demand. With the emergence of smart grid, it is possible for the grid operator to communicate with the users and try to incentivize the users to shift load from heavy load regions to light load regions. In our proposed framework, the smart grid optimizes dynamic prices by setting the charging thresholds $s$ to achieve the power load balancing. To measure the power load levels in different locations, we define the electric load ratio in location $i$ at time $t$ as

$$
r_{i}^{t}(s)=\frac{E_{i}^{t}(\mathbf{s})+B_{i}^{t}}{C_{i}}
$$

where $E_{i}^{t}$ is the energy consumption of data center $i$ and in general depends on the energy threshold $s$ for all locations and all time slots, ${ }^{1} B_{i}^{t}$ is the background power load, and $C_{i}$ is the capacity of power substation $i$. In general, if the load ratio is high, then the grid operator can better utilize the installed capacity. However, high load ratio may also increase the risk of overloading the power system.

Let $q_{i}^{t}=C_{i}-B_{i}^{t}$ be the maximum available power supply to the data center $i$ at time $t$. Usually, conventional electricity users have inflexible usage patterns and their demands can be inelastic. However, data centers have a very good flexibility in energy consumption because of the ability of routing workload among different locations. Therefore, we denote the total energy usage of conventional users other than data centers as the background energy load $B_{i}^{t}$. Since our study focuses on the impact of data center energy consumption, we assume the background energy load can be predicted accurately [18].

Based on the load ratio $r_{i}^{t}$, we define the electric load index $(E L I)$ across all locations and time slots as

$$
E L I \triangleq \sum_{t \in \mathcal{T}} \sum_{i \in \mathcal{N}}\left(r_{i}^{t}(s)\right)^{2} C_{i},
$$

where $E L I$ is motivated by the index measurement techniques $[19,20]$ used for feeder load balancing in distribution system. ELI not only measures the overall load ratio but also gives different weights based on the capacities in different locations. The smart grid aims at balancing the load ratio $r_{i}^{t}(\boldsymbol{s})$ at all locations and all time slots by minimizing the ELI. We will show later that minimizing ELI results in balancing the electric load across all the locations.

To balance the electric power load, the grid operator provides rewards (price discounts) to incentivize users to shift their electricity usage. As power grid is usually a regulated industry, we assume that the regulator sets the upper bound and lower bound of the charging threshold $s$ as follows.

First, the unit price $\pi_{i}^{t}$ defined in (1) should be nonnegative, hence

$$
\alpha_{i}^{t}+\beta_{i}\left(E_{i}^{t}-s_{i}^{t}\right) \geq 0 .
$$

Second, the unit price should be lower than the LMP $\alpha_{i}^{t}$, since we assume that smart grid only provides price discount (instead of charging extra) to data centers. This means

$$
\alpha_{i}^{t}+\beta_{i}\left(E_{i}^{t}-s_{i}^{t}\right) \leq \alpha_{i}^{t} .
$$

${ }^{1}$ In Section 3, we will show that our two-stage problem can be decomposed across time, and hence $E_{i}^{t}$ only depends on the thresholds in time slot $t$. 
Combining (9) and (10), we get the constraints for $\boldsymbol{s}$ :

$$
E_{i}^{t} \leq s_{i}^{t} \leq \frac{\alpha_{i}^{t}}{\beta_{i}}+E_{i}^{t}, \forall i \in \mathcal{N}, t \in \mathcal{T},
$$

where the upper bound $\frac{\alpha_{i}^{t}}{\beta_{i}}+E_{i}^{t}$ and lower bound $E_{i}^{t}$ are not constant, but functions of $\boldsymbol{s}$. We will solve $E_{i}^{t}$ explicitly in Section 3. Hence, (11) are not box constraints but are general inequalities.

The ELI is not only a technical measure, but also an economic indicator. The higher ELI is, the more costly to maintain the stability of the power system. Furthermore, if load ratio $r_{i}^{t}$ is close to $100 \%$, then the grid operator must invest more in electricity facilities to prevent the demand from exceeding the capacity, and such capacity investment is extremely costly. Meanwhile, we also note that the smart grid needs to give discounts to the users (through a proper choice of $\boldsymbol{s}$ ) to achieve load balancing, and such discounts lead to the grid operator's revenue loss. Therefore, the smart grid will aim at minimizing the weighted sum of the cost represented by ELI and revenue loss caused by offering discounts to the data centers. ${ }^{2}$

Stage 1: Electric power load balancing

$$
\min _{\boldsymbol{s}} \sum_{t \in \mathcal{T}} \sum_{i \in \mathcal{N}} \theta C_{i}\left(r_{i}^{t}(\boldsymbol{s})\right)^{2}+(1-\theta) \beta_{i}\left(s_{i}^{t}-E_{i}^{t}(\boldsymbol{s})\right) E_{i}^{t}(\boldsymbol{s})
$$

subject to Constraint (11),

where $\theta \in[0,1]$ is a tradeoff coefficient.

\section{TWO-STAGE PROBLEM}

We will solve the problem through backward induction. We first solve the Stage-2 problem, where data centers minimize the total energy cost. Then we design a branch and bound algorithm for the Stage-1 problem to attain the globally optimal solution.

\subsection{Solving the Stage-2 problem}

In the Stage-2 problem, data centers decide the workload allocation $\lambda_{i}^{t}$ and number of active servers $x_{i}^{t}$ at all locations and all time slots to minimize the total energy cost. We will first decompose the workload allocation problem to $T$ equivalent problems shown in Lemma 1 , and solve the optimal energy consumption $E_{i}^{t}$ of each data center $i$ at each time slot $t$.

Lemma 1. Solving the Stage-2 problem (PS2) is equivalent of solving $T$ subproblems separately, where each subprogram involves the energy consumption distribution in a time slot $t \in \mathcal{T}$ as follows.

$$
\begin{array}{ll}
\min _{E_{i}^{t}, \forall i \in \mathcal{N}} & \sum_{i \in \mathcal{N}}\left(\alpha_{i}^{t}+\beta_{i}\left(E_{i}^{t}-s_{i}^{t}\right)\right) E_{i}^{t} \\
\text { subject to } & \sum_{i \in \mathcal{N}} E_{i}^{t}=E^{t}, \\
& \underline{\phi_{i}^{t}} \leq E_{i}^{t} \leq \overline{\phi_{i}^{t}}, \forall i \in \mathcal{N} .
\end{array}
$$

where $E^{t}$ is the total energy consumption needed in time slot $t$ to process the total workload $L^{t}$, and $\phi_{i}^{t}$ and $\overline{\phi_{i}^{t}}$ are the minimum and maximum energy consumptions, respectively. Here $E^{t}=\left(a+\frac{b}{\mu}\right) L^{t}+\sum_{i \in \mathcal{N}}\left(\frac{b}{\mu\left(D-d_{i}^{t}\right)}+c\right), \underline{\phi_{i}^{t}}=\frac{b}{\mu\left(D-d_{i}^{t}\right)}+c$, and $\overline{\phi_{i}^{t}}=\min \left\{q_{i}^{t}, a\left(\mu M_{i}-\frac{1}{D-d_{i}^{t}}\right)+b M_{i}+c\right\}$. The readers can find the proof of Lemma 1 in [28].

${ }^{2}$ If we allow the grid operator to freely choose the prices (i.e., higher or lower than LMP), then the ELI maybe further reduced comparing with the case of providing price discounts only. Hence our proposed scheme provides a performance lower-bound for the grid operator in practice.
As Problem (PE2-t) is strictly convex, we can compute the optimal solution $E_{i}^{t *}$ through the Lagrangian dual method. This leads to the following result.

TheOREM 1. The unique optimal solution of Problem (PE2t) is

$$
E_{i}^{t *}=\operatorname{mid}\left(\underline{\phi_{i}^{t}}, \frac{s_{i}^{t}}{2}-\frac{\alpha_{i}^{t}+\sigma^{t}}{2 \beta_{i}}, \overline{\phi_{i}^{t}}\right), \forall i \in \mathcal{N}
$$

where $\sigma^{t}$ is the Lagrangian multiplier corresponding to the equality constraint $\sum_{i \in \mathcal{N}} E_{i}^{t}=E^{t}$, and the operator mid means the median of its three arguments.

Problem (PE2-t) can be solved by the standard subgradient method with constant stepsize [21], shown in [28].

\subsection{Solving the Stage-1 problem}

After solving the Stage-2 problem, we obtain the optimal energy consumption of data centers as functions (12) of the given charging thresholds $\boldsymbol{s}$. As a quadratic bilevel program, solving the Stage-1 problem (PS1) is NP-hard [22]. However, we notice that solving (PS1) is equivalent to solving $T$ subproblems in each time slot $t$ separately. We denote the subproblem in time slot $t$ as (PS1-t) and write (PS1-t) as

$$
\begin{aligned}
& \min _{\left\{s_{i}^{t}, E_{i}^{t}, \sigma^{t}, \underline{\omega_{i}^{t}}, \overline{\omega_{i}^{t}}\right\}_{\forall i \in \mathcal{N}}} \sum_{i \in \mathcal{N}} \theta C_{i}\left(r_{i}^{t}\right)^{2}+(1-\theta) \beta_{i}\left(s_{i}^{t}-E_{i}^{t}\right) E_{i}^{t} \\
& \text { subject to } E_{i}^{t} \leq s_{i}^{t} \leq \frac{\alpha_{i}^{t}}{\beta_{i}}+E_{i}^{t}, \forall i \in \mathcal{N} \\
& \alpha_{i}^{t}+2 \beta_{i} E_{i}^{t}-\beta_{i} s_{i}^{t}+\sigma^{t}-\underline{\omega_{i}^{t}}+\overline{\omega_{i}^{t}}=0, \forall i \in \mathcal{N}, \\
& \underline{\omega}_{i}^{t}\left(\underline{\phi_{i}^{t}}-E_{i}^{t}\right)=0, \forall i \in \mathcal{N} \\
& \bar{\omega}_{i}^{t}\left(E_{i}^{t}-\overline{\phi_{i}^{t}}\right)=0, \forall i \in \mathcal{N} \text {, } \\
& \sum_{i \in \mathcal{N}} E_{i}^{t}=E^{t} \\
& \underline{\phi_{i}^{t}} \leq E_{i}^{t} \leq \overline{\phi_{i}^{t}}, \forall i \in \mathcal{N} \\
& \underline{\omega_{i}^{t}} \geq 0, \overline{\omega_{i}^{t}} \geq 0, \quad \forall i \in \mathcal{N},
\end{aligned}
$$

where (14)-(19) are the KKT conditions of the Stage-2 problem, and $\sigma^{t}, \omega_{i}^{t}$, and $\overline{\omega_{i}^{t}}$ are the Lagrange multipliers associated with the equality and box constraints of Problem (PE2$\mathrm{t}$ ). The equivalence of the KKT formulation is guaranteed because Problem (PE2-t) is strictly convex and hence the KKT conditions are necessary and sufficient for the globally optimal solution of Problem (PE2-t).

To linearize the complementarity slackness conditions (15) and (16), we introduce binary variables $\underline{z}_{i}^{t}$ and $\bar{z}_{i}^{t}$, and replace (15) and (16) by the following constraints.

$$
\begin{aligned}
& E_{i}^{t}-\underline{\phi}_{i}^{t} \leq \underline{z}_{i}^{t} M, \forall i \in \mathcal{N}, \\
& \underline{\omega}_{i}^{t} \leq\left(1-\underline{z}_{i}^{t}\right) M, \forall i \in \mathcal{N}, \\
& \overline{\phi_{i}^{t}}-E_{i}^{t} \leq \bar{z}_{i}^{t} M, \forall i \in \mathcal{N}, \\
& \bar{\omega}_{i}^{t} \leq\left(1-\bar{z}_{i}^{t}\right) M, \forall i \in \mathcal{N},
\end{aligned}
$$

where $M$ is a sufficiently large constant.

We observe that the objective of Problem (PS1-t) is an indefinite quadratic function, hence it is NP-hard [23]. Since the nonconvexity only comes from the indefinite quadratic objective, we can reformulate the problem and design a branch and bound algorithm to solve (PS1-t) and attain the globally optimal solution [23]. There are two advantages of using the branch and bound algorithm to solve our problem. The first one is that the branch and bound algorithm incorporates the KKT conditions in our problem, where the branching rule is designed based on the complementarity slackness conditions. The second one is that the branch and bound tree of our problem is finite, which guarantees the convergence of the algorithm [24]. Denote 
$\boldsymbol{E}^{t}=\left\{E_{i}^{t}, \forall i \in \mathcal{N}\right\}, \boldsymbol{s}^{t}=\left\{s_{i}^{t}, \forall i \in \mathcal{N}\right\}, \underline{\boldsymbol{\omega}}^{t}=\left\{\omega_{i}^{t}, \forall i \in \mathcal{N}\right\}$, and $\overline{\boldsymbol{\omega}}^{t}=\left\{\overline{\omega_{i}^{t}}, \forall i \in \mathcal{N}\right\}$, we can rewrite Problem (PS1-t) as the general form noted as (PE1-t).

$$
\begin{aligned}
\min _{\boldsymbol{E}^{t}, \boldsymbol{s}^{t}, \sigma^{t}, \underline{\boldsymbol{\omega}}^{t}, \overline{\boldsymbol{\omega}}^{t}} & \frac{1}{2} \boldsymbol{E}^{t T} \boldsymbol{H}_{1} \boldsymbol{E}^{t}+\boldsymbol{s}^{t T} \boldsymbol{H}_{2} \boldsymbol{E}^{t}+\boldsymbol{f}^{t T} \boldsymbol{E}^{t}+\boldsymbol{B}^{t T} \boldsymbol{H}_{3} \boldsymbol{B}^{t} \\
\text { subject to } & \boldsymbol{E}^{t}-\boldsymbol{s}^{t} \leq \mathbf{0}, \quad \boldsymbol{s}^{t}-\boldsymbol{E}^{t} \leq \boldsymbol{b}_{1}^{t}, \\
& 2 \boldsymbol{H}_{4} \boldsymbol{E}^{t}-\boldsymbol{H}_{4} \boldsymbol{s}^{t}+\sigma^{t} \mathbf{1}-\underline{\boldsymbol{\omega}}^{t}+\overline{\boldsymbol{\omega}}^{t}=\boldsymbol{b}_{2}^{t}, \\
& \mathbf{1}^{T} \boldsymbol{E}^{t}=E^{t}, \\
& \boldsymbol{b}_{3}^{t}-\boldsymbol{E}^{t} \leq \mathbf{0}, \quad \boldsymbol{E}^{t}-\boldsymbol{b}_{4}^{t} \leq \mathbf{0}, \\
& \underline{\boldsymbol{\omega}}^{t} \geq \mathbf{0}, \quad \underline{\boldsymbol{\omega}}^{t} \leq \boldsymbol{b}_{5}^{t}, \\
& \overline{\boldsymbol{\omega}}^{t} \geq \mathbf{0}, \quad \overline{\boldsymbol{\omega}}^{t} \leq \boldsymbol{b}_{6}^{t},
\end{aligned}
$$

where the detailed formulation including the parameter matrices and vectors are presented in [28] due to space limit.

Then we reformulate the general quadratic programming problem (PE1-t) as a linear programming problem with complementarity constraints [23]. Let us introduce multipliers $\boldsymbol{v}_{1}^{t} \geq \mathbf{0}, \boldsymbol{v}_{2}^{t} \geq \mathbf{0}, \boldsymbol{v}_{3}^{t}, v_{4}^{t}, \boldsymbol{v}_{5}^{t} \geq \mathbf{0}, \boldsymbol{v}_{6}^{t} \geq \mathbf{0}, \boldsymbol{v}_{7}^{t} \geq \mathbf{0}, \boldsymbol{v}_{8}^{t} \geq \mathbf{0}$, $\boldsymbol{v}_{9}^{t} \geq \mathbf{0}$, and $\boldsymbol{v}_{10}^{t} \geq \mathbf{0}$, associated with the inequality, equality and box constraints of Problem (PE1-t), where $v_{4}^{t} \in \mathbb{R}$, and $\boldsymbol{v}_{j}^{t} \in \mathbb{R}^{N \times 1}, j \in\{1, \ldots, 10\} \backslash\{4\}$. Given any locally optimal solution of Problem (PE1-t), we can define the following two sets for the multipliers

$\mathcal{G}=\left\{\boldsymbol{H}_{2} \boldsymbol{E}^{t}-\boldsymbol{v}_{1}^{t}+\boldsymbol{v}_{2}^{t}-\boldsymbol{H}_{4} \boldsymbol{v}_{3}^{t}=\mathbf{0}\right.$,

$\boldsymbol{H}_{1} \boldsymbol{E}^{t}+\boldsymbol{H}_{2} \boldsymbol{s}^{t}+\boldsymbol{f}^{t}+\boldsymbol{v}_{1}^{t}-\boldsymbol{v}_{2}^{t}+2 \boldsymbol{H}_{4} \boldsymbol{v}_{3}^{t}+v_{4}^{t} \mathbf{1}-\boldsymbol{v}_{5}^{t}+\boldsymbol{v}_{6}^{t}=\mathbf{0}$

$\left.\mathbf{1}^{T} \boldsymbol{v}_{3}^{t}=0,-\boldsymbol{v}_{3}^{t}-\boldsymbol{v}_{7}^{t}+\boldsymbol{v}_{8}^{t}=\mathbf{0}, \boldsymbol{v}_{3}^{t}-\boldsymbol{v}_{9}^{t}+\boldsymbol{v}_{10}^{t}=\mathbf{0}\right\}$,

$\mathcal{C}=\left\{\left(\boldsymbol{E}^{t}-\boldsymbol{s}^{t}\right) \circ \boldsymbol{v}_{1}^{t}=\mathbf{0},\left(\boldsymbol{s}^{t}-\boldsymbol{E}^{t}-\boldsymbol{b}_{1}^{t}\right) \circ \boldsymbol{v}_{2}^{t}=\mathbf{0}\right.$,

$\left(\boldsymbol{b}_{3}^{t}-\boldsymbol{E}^{t}\right) \circ \boldsymbol{v}_{5}^{t}=\mathbf{0},\left(\boldsymbol{E}^{t}-\boldsymbol{b}_{4}^{t}\right) \circ \boldsymbol{v}_{6}^{t}=\mathbf{0}, \underline{\boldsymbol{\omega}}^{t} \circ \boldsymbol{v}_{7}^{t}=\mathbf{0}$,

$\left.\left(\underline{\boldsymbol{\omega}}^{t}-\boldsymbol{b}_{5}^{t}\right) \circ \boldsymbol{v}_{8}^{t}=\mathbf{0}, \overline{\boldsymbol{\omega}}^{t} \circ \boldsymbol{v}_{9}^{t}=\mathbf{0},\left(\overline{\boldsymbol{\omega}}^{t}-\boldsymbol{b}_{6}^{t}\right) \circ \boldsymbol{v}_{10}^{t}=\mathbf{0}\right\}$,

where $\mathcal{G}$ is the set of Lagrange stationarity conditions, $\mathcal{C}$ consists of those multipliers satisfying complementarity slackness conditions, and "o" denotes the component-wise product of two vectors. According to [23], if $\mathcal{G} \cap \mathcal{C} \neq \emptyset$, then the KKT conditions are necessary for a local (and also a global) optimal solution.

THEOREM 2. (Reformulation) The Stage-1 problem (PE1t) can be written in the following equivalent linear programming with complementarity constraints, denoted as (PR1-t).

$$
\begin{aligned}
\min & \frac{1}{2} \boldsymbol{f}^{t T} \boldsymbol{E}^{t}-\frac{1}{2} \boldsymbol{b}_{1}^{t T} \boldsymbol{v}_{2}^{t}+\frac{1}{2}\left(-\boldsymbol{b}_{2}^{t}+\sigma^{t} \mathbf{1}-\underline{\boldsymbol{\omega}}^{t}+\overline{\boldsymbol{\omega}}^{t}\right)^{T} \boldsymbol{v}_{3}^{t} \\
& -\frac{1}{2} E^{t} v_{4}^{t}+\frac{1}{2} \boldsymbol{b}_{3}^{t T} \boldsymbol{v}_{5}^{t}-\frac{1}{2} \boldsymbol{b}_{4}^{t T} \boldsymbol{v}_{6}^{t}+\boldsymbol{B}^{t T} \boldsymbol{H}_{3} \boldsymbol{B}^{t}(P R 1-t)
\end{aligned}
$$

subject to $(24)-(29), \boldsymbol{v}_{j}^{t}, v_{4}^{t} \in \mathcal{G} \cap \mathcal{C}, j \in\{1, \ldots, 10\} \backslash\{4\}$,

where $\boldsymbol{B}^{t T} \boldsymbol{H}_{3} \boldsymbol{B}^{t}$ is a constant. The problem (PR1-t) is nonconvex, however, the nonconvex terms only come from the complementarity slackness conditions $\mathcal{C}$ and binary variables $\underline{z}_{i}^{t}, \bar{z}_{i}^{t}$, which can be enforced by using linear equations, e.g., either $\left(\boldsymbol{E}^{t}-\boldsymbol{s}^{t}\right)_{i}=0$ or $\boldsymbol{v}_{1 i}^{t}=0,\left(\boldsymbol{s}^{t}-\boldsymbol{E}^{t}-\boldsymbol{b}_{1}^{t}\right)_{i}=0$ or $\boldsymbol{v}_{2 i}^{t}=0,\left(\boldsymbol{b}_{3}^{t}-\boldsymbol{E}^{t}\right)_{i}=0$ or $\boldsymbol{v}_{5 i}^{t}=0,\left(\boldsymbol{E}^{t}-\boldsymbol{b}_{4}^{t}\right)_{i}=0$ or $\boldsymbol{v}_{6 i}^{t}=0$, $\underline{\boldsymbol{\omega}}_{i}^{t}=0$ or $\boldsymbol{v}_{7 i}^{t}=0,\left(\underline{\boldsymbol{\omega}}^{t}-\boldsymbol{b}_{5}^{t}\right)_{i}=0$ or $\boldsymbol{v}_{8 i}^{t}=0, \overline{\boldsymbol{\omega}}_{i}^{t}=0$ or $\overline{\boldsymbol{v}}_{9 i}^{t}=0,\left(\overline{\boldsymbol{\omega}}^{t}-\boldsymbol{b}_{6}^{t}\right)_{i}=0$ or $\boldsymbol{v}_{10 i}^{t}=0, \underline{z}_{i}^{t}=0$ or $\underline{z}_{i}^{t}=1, \bar{z}_{i}^{t}=0$ or $\bar{z}_{i}^{t}=1$, where $i \in \mathcal{N}$ denotes the $i$ th component.

By dropping the complementarity slackness conditions $\mathcal{C}$ and binary constraints from (PR1-t), we obtain the relaxed LP problem (RLP1-t).

$$
\begin{aligned}
\min & \frac{1}{2} \boldsymbol{f}^{t T} \boldsymbol{E}^{t}-\frac{1}{2} \boldsymbol{b}_{1}^{t T} \boldsymbol{v}_{2}^{t}+\frac{1}{2}\left(-\boldsymbol{b}_{2}^{t}+\sigma^{t} \mathbf{1}-\underline{\omega}^{t}+\overline{\boldsymbol{\omega}}^{t}\right)^{T} \boldsymbol{v}_{3}^{t} \\
& -\frac{1}{2} E^{t} v_{4}^{t}+\frac{1}{2} \boldsymbol{b}_{3}^{t T} \boldsymbol{v}_{5}^{t}-\frac{1}{2} \boldsymbol{b}_{4}^{t T} \boldsymbol{v}_{6}^{t}+\boldsymbol{B}^{t T} \boldsymbol{H}_{3} \boldsymbol{B}^{t}(R L P 1-t)
\end{aligned}
$$

subject to $(24)-(29), \boldsymbol{v}_{j}^{t}, v_{4}^{t} \in \mathcal{G}, j \in\{1, \ldots, 10\} \backslash\{4\}$.
We use branch and bound algorithm [22] to solve (PR1-t). Starting from the relaxed LP problem (RLP1-t), we can add the linear constraint, e.g., $\left(\boldsymbol{E}^{t}-\boldsymbol{s}^{t}\right)_{i}=0$ or $\boldsymbol{v}_{1 i}^{t}=0$, then we get two new LP problems (e.g., two first level children nodes in the branch and bound tree). We can continue to expand the tree by adding other linear constraints until $\mathcal{C}$ is completely enforced. The LP problem in each node can be solved by the interior-point method efficiently [21], and the optimal objective value to the LP problem sets an lowerbound of the optimal objective value of the original problem (PR1-t). Any feasible solution provides an upperbound. The branch and bound algorithm terminates at a globally optimal solution when the lowerbound meets the upperbound or all the nodes in the branch and bound tree have been evaluated [23]. The worst-case complexity is $\mathcal{O}\left(2^{10 N}\right)$.

\section{SIMULATION RESULTS}

In this section, we evaluate our proposed algorithm based on realistic system parameters, compare the electric load index between the integrated model and the two-stage model, and compare the energy cost of data centers with LMPbased pricing and dynamic pricing. In the integrated problem, smart grid operator directly controls the workload assignments of the data centers, and can achieve the best load balancing performance. We run our simulations for four data centers geographically located in four different regions: New York, Maine, Rhode Island, and Boston. We took hourly locational marginal prices and demands of the four locations on 4th March 2013 as the base prices and background power load in our experiment $[25,26]$. The dynamic computing requests are simulated based on the Worldcup98 workload trace [27]. For other simulation parameters, please refer to our technical report [28].

We first evaluate the performance of load balancing in smart grid. The evolution of electric load index of three scenarios over 24 hours is shown in Figure 3. The black curve represents the $E L I$ of the integrated solution, and the red curve represents the $E L I$ of the optimal dynamic solution of the two-stage problem. The red curve is very close to the black curve, which suggests the optimal two-stage dynamic pricing achieves the performance very close to the best possible load balancing. ${ }^{3}$ The blue curve represents the LMP benchmark, where the data centers are charged based on the location-dependent and time-dependent LMP pricing. Figure 3 shows that our proposed scheme reduces the ELI by around $12 \%$ comparing with the LMP benchmark.

The evolution of data centers' total energy cost over 24 hours is depicted in Figure 4. The energy cost with dynamic pricing is less than the LMP benchmark, as the dynamic pricing only offers discounts from the LMP prices, which implies that data centers reduce the total energy cost (across 24 hours) by $46 \%$ by taking advantage of the dynamic price discounts and reallocating the workload. From Figure 3 and Figure 4, we observe that the dynamic interactions between smart grid and data centers bring benefits to both sides and achieve a win-win situation.

We now examine the power load distribution within one particular hour (e.g., hour 20), and plot the background power load, power load of data centers, and the total load. Figure 5 shows the electric load distribution in the two-stage model. We can see the smart grid tries to drive the load evenly distributed with the same usage ratio among all the locations. Since location 1 has high background load, the

${ }^{3}$ The ELI performance of the two-stage problem is related to the configuration of coefficient $\theta$ in (PS-1). In our simulations, we set $\theta$ quite close to 1 , which explains why the performance is close to optimal. In our future work, we intend to further explore the impact of changing $\theta$. 

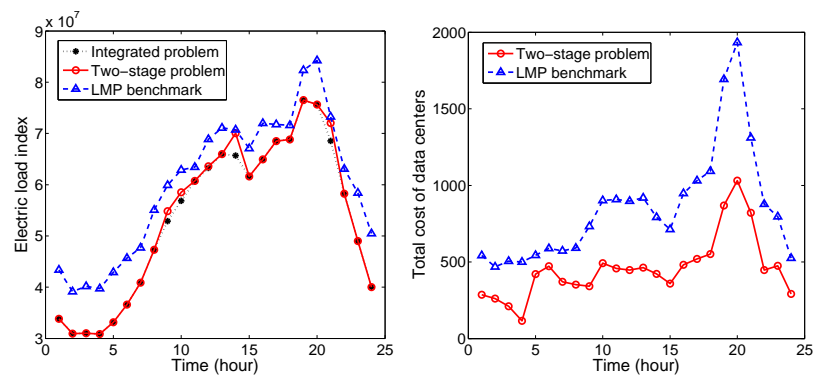

Figure 3: Electric load index of smart grid

Figure 4: Total energy cost of data centers

data center in location 1 only consumes the minimum energy to avoid overload. Since the location 2 and location 3 have the same capacity $30 \mathrm{MW}$, the total loads are the same in location 2 and location 3. However, in Figure 6, the energy usage is not balanced, since in the LMP-based pricing scenario, data centers assign workload to the location with the lowest LMP price as much as possible to minimize the energy cost. The consequence is that the power load is extremely high in the lowest price location 1, bringing a risk of blackout. Therefore, our proposed scheme can effectively enhance the robustness of power grid through load balancing.

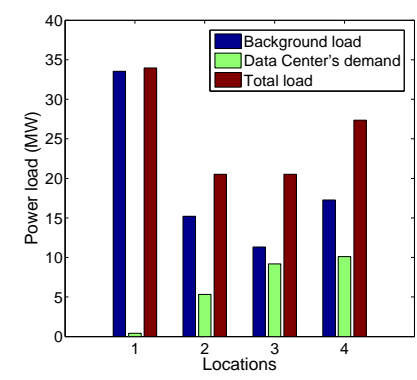

Figure 5: Power load (Two-stage optimal dynamic pricing)

\section{CONCLUSION}

In this paper, we studied the dynamic interactions between smart grid and data centers as a two-stage problem. To solve the two-stage optimization problem, we reformulated it as a linear programming problem with complementarity slackness conditions, and proposed a branch and bound algorithm to attain the globally optimal solution. The simulation experiments showed the electric load index is decreased by around $12 \%$, and the total energy cost of data centers is reduced by $46 \%$ on average.

In the future, we plan to investigate some approximation and heuristic algorithms to further reduce the complexity of solving the two-stage problem. We will also consider the impact of renewable energy, energy storage, grid topology, and other operating costs of data centers (such as the server switching cost) in the study of smart grid and data center interactions.

\section{REFERENCES}

[1] A. Qureshi, R. Weber, H. Balakrishnan, J. Guttag, and B. Maggs, "Cutting the electric bill for Internet-scale systems," in Proc. of ACM SIGCOMM, 2009.

[2] M. Pedram, "Energy-efficient datacenters," IEEE Trans. on Computer Aided Design, 31(10), 2012.

[3] Z. Liu, M. Lin, A. Wierman, S.H. Low, and L.L.H. Andrew, "Greening geographical load balancing," in Proc. of ACM SIGMETRICS, 2011.

[4] Z. Liu, M. Lin, A. Wierman, S.H. Low, and L.L.H. Andrew, "Geographical load balancing with renewables," in Proc. of ACM Greenmetrics, 2011.
[5] Z. Liu, A. Wierman, Y. Chen, and B. Razon, "Data center demand response: avoiding the coincident peak via workload shifting and local generation," in Proc. of ACM SIGMETRICS, 2013.

[6] H. Xu, C. Feng, and B. Li. "Temperature aware workload management in geo-distributed datacenters," in Proc. of ICAC, 2013.

[7] L. Rao, X. Liu, L. Xie, and W. Liu, "Minimizing electricity cost: optimization of distributed Internet data centers in a multi-electricity-market environment,' in Proc. of IEEE INFOCOM, 2010.

[8] P. Wang, L. Rao, X. Liu, and Y. Qi, "D-pro: dynamic data center operations with demand-responsive electricity prices in smart grid," IEEE Trans. on Smart Grid, 3(4): 1743-1754, 2012.

[9] A.H. Mohsenian-Rad, and A. Leon-Garcia, "Coordination of cloud computing and smart power grids," in Proc. of IEEE SmartGridComm, 2010.

[10] X. Fang, S. Misra, G. Xue, and D. Yang, "Smart grid-the new and improved power grid: a survey," IEEE Communications Surveys and Tutorials, 14: 944-980, 2012.

[11] A.H. Mohsenian-Rad, and A. Leon-Garcia, "Optimal residential load control with price prediction in real-time electricity pricing environments," IEEE Trans. on Smart Grid, 1(2): 120-133, 2010.

[12] A.H. Mohsenian-Rad, V.W.S. Wong, J. Jatskevich, R. Schober, and A. Leon-Garcia, "Autonomous demand-side management based on game-theoretic energy consumption scheduling for the future smart grid," IEEE Trans. on Smart Grid, 1(3): 320-331, 2010

[13] H.K. Nguyen, J.B. Song, and Z. Han, "Demand side management to reduce peak-to-average ratio using game theory in smart grid," in Proc. of IEEE INFOCOM Workshops, 2012.

[14] C. Chen, S. Kishore, and L.V. Snyder, "An innovative RTP-based residential power scheduling scheme for smart grids," in Proc. of IEEE ICASSP, 2011.

[15] C.J. Wong, S. Ha, S. Sen, and M. Chiang, "Optimized day-ahead pricing for the smart grid with device-specific scheduling flexibility," IEEE Journal on Selected Areas in Communications, 1075-1085, 2012.

[16] D. Kirschen, and G. Strbac, "Fundamentals of power system economics," Wiley, 2004.

[17] J. Lai, et al, "Evaluation of evolving residential electricity tariffs," Ernest Orlando Lawrence Berkeley National Laboratory, 2011.

[18] A. Motamedi, H. Zareipour and W.D. Rosehart, "Electricity price and demand forecasting in smart grids," IEEE Trans. on Smart Grid, 3(2): 664-674, 2012 .

[19] W.M. Lin, and H.C. Chin, "A current index based load balance technique for distribution systems," in Proc. of PowerCon, 1998.

[20] M.A. Kashem, and V. Ganapathy, "Three-phase load balancing in distribution systems using index measurement technique," International Journal of Electrical Power \& Energy Systems, 24(1), 31-40, 2002.

[21] S. Boyd, and L. Vandenberghe, Convex Optimization. Cambridge University Press, 2004.

[22] J.F. Bard, "Practical bilevel optimization: algorithms and applications," Kluwer Academic Publishers, 1998.

[23] S. Burer, and D. Vandenbussche, "A finite branch-and -bound algorithm for nonconvex quadratic programm -ing via semidefinite relaxations," Mathematical Programming: Series A, 113(2): 259-282, 2008.

[24] J. Chen, and S. Burer, "Globally solving nonconvex quadratic programming problems via completely positive programming," Mathematical Programming Computation, 4(1): 33-52, 2012.

[25] http://www.iso-ne.com

[26] http://www.nyiso.com

[27] http://ita.ee.lbl.gov/html/contrib/WorldCup.html

[28] H. Wang, J. Huang, X. Lin, and A.H. Mohsenian-Rad, Technical Report. [Online] http://jianwei.ie.cuhk.edu.hk/publication/ SmartGridDataCenterInteractionGCReport.pdf 